\title{
Étude expérimentale du comportement de groupe de pieux chargés latéralement et de l'effet de leur mise en place dans un massif sableux
}

\section{S. MEZAZIGH}

D. LEVACHER

Universite du Havre, Laboratoire de Mécanique, GRGC,

Quai Frissard, BP 265, 76055 Le Havre Cedex

\section{J. GARNIER}

Laboratoire central des Ponts et Chaussées de Nantes, BP 19, 44340 Bouguenais
La plupart des recherches sur les pieux sollicités latéralement ont été menées sur des pieux isolés mais les pieux sont fréquemment utilisés en groupe, et peu d'informations sont disponibles sur l'effet d'interaction pieu-sol-pieu et sur la performance du groupe de pieux. Le problème est tridimensionnel ,et l'interaction sol-pieu est trop complexe pour que cette étude puisse être conduite à l'aide de méthodes théoriques ou numériques. Compte tenu du nombre d'essais nécessaires, elle ne peut guère être réalisée à l'aide d'essais sur des pieux réels. Une étude a ainsi été entreprise sur modèles centrifugés pour déterminer l'influence de la disposition des pieux en groupe et leur mode de mise en place sur leur comportement. On présente dans cet article les résultats de 40 essais de couple de pieux chargés latéralement

Mots clés: pieu, charge horizontale, groupe de pieux, modèles réduits centrifugés, déplacements, moments fléchissants, courbes de réaction.

Experimental study of laterally loaded pile group behaviour and their installation effect in sand

The investigations and researches on laterally loaded piles are concerning single piles, but piles are commoniy used in groups. and few informations are available on pile-sol-pile interaction effect and on the laterally loaded piles group performance. The problem is very complex to be study with theoretical or numerical methods. As a large number of tests are necessary, they could not to be run on true scale piles.

However, with small scale model tests, it is important to make the simulation as perfectly as possible, either in reversible domain or in rupture domain. The mass forces simulations by centrifuge is well adapted to answer to this kind of problem. All tests have been done at the Laboratoire des Ponts et Chaussées of Nantes to determine the group and the installation effect on the behavior of the laterally loaded pile. In this paper, the analysis results of 40 tests of laterally loaded couples of piles in centrifuge are presented and discussed.

Key words: pile, horizontal loads, group effects, models. centrifuge, displacement, bending moment, reaction curves. 


\section{Notations}

B: diamètre du pieu (m).

e: efficacité du groupe (m).

FH: effort horizontal (kN).

L: fiche du pieu dans le sol (m).

S: espacement entre axe des pieux du couple (m).

$\mathrm{z}$ : profondeur dans le sol (m).

P: pression latérale $\left(\mathrm{kN} / \mathrm{m}^{2}\right)$.

$\mathrm{Y}$ : déplacement latérale $(\mathrm{m})$.

\section{1}

\section{Introduction}

Le recours aux pieux inclinés a longtemps été la solution préconisée pour la reprise de charges horizontales sur les fondations profondes.

Des innovations technologiques qui concernent la méthode de mise en ceuvre, l'utilisation de nouveaux matériaux, le contrôle et la qualité de la réalisation ont permis de trouver une alternative à l'utilisation des pieux inclinés dont l'exécution reste délicate. De même, une meilleure compréhension des mécanismes qui régissent le comportement des fondations profondes soumises à des chargements latéraux a suscité le développement de nombreuses méthodes de dimensionnement qui n'ont fait que renforcer, dans le monde de la construction, l'idée d'utiliser les pieux verticaux pour reprendre aussi les charges horizontales.

Si certaines méthodes de calcul sont couramment utilisées (méthode basées sur la théorie de l'èlasticité et sur la notion de courbes de réaction), les méthodes numériques encore sujet de discussions devant la difficulté de déterminer les paramètres qui doivent traduire le comportement mécanique du sol au voisinage du pieu (lois d'interface) et en zone éloignée. En outre, dans la pratique, le problème du pieu isolé implanté dans un sol à surface horizontale reste un cas idéal assez rare (pieu d'accostage ou d'amarrage). Dans la réalité, les pieux sont associès en files, en rangées ou en groupes dont les comportements sous charges latérales deviennent très complexes (interaction, efficacíté, installation). Les méthodes de dimensionnement restent encore très grossières et des recherches ont été engagées sur ce sujet dans le réseau des laboratoires des Ponts et Chaussées. A cela vient s'ajouter l'environnement du pieu : terrains en pente ou à proximité de pentes (souilles, talus) et constructions existantes (remblais, ouvrages en terre, bàtiment...).

Les essais sur modèles réduits centrifugés constituent l'une des approches retenues car ils présentent les mêmes avantages que les essais sur modèles de laboratoire (répétitivité, contrôle rigoureux des conditions expérimentales, études paramétriques possibles, étalonnage de la réponse des pieux instrumentés avant essai...). En outre, du fait du respect de certaines conditions de similitude et en particulier des forces de masses, leurs résultats peuvent être transposés aux ouvrages en vraie grandeur, ce qui n'est pas le cas des modèles de laboratoire (Garnier, 1995).

Par ailleurs, un des paramètres importants qui n'est pas pris en considération et qui entraîne des difficultés dans la prévision du comportement d'un groupe de pieux sollicité latéralement, est le remaniement du sol induit lors de la mise en place du groupe, plus particulièrement s'il s'agit de pieux refoulant le sol. En effet les travaux de José et al, (1991) utilisant une analyse simplifiée d'un problème axisymétrique, ont montré l'influence de l'installation des pieux en groupe dans un sable, sur les déplacements et les contraintes horizontales dans le sol. Les auteurs recommandent alors une distance minimale égale à $15 R$ où $R$ est le rayon du pieu, au-delà de laquelle l'interaction entre les pieux du groupe peut être négligée. D'autres auteurs se sont aussi intéressés au problème d'installation de pieux isolés et de groupe de pieux et on montré l'importance de la perturbation résultant de la mise en place (Cooke et al., 1979; Shublaq, 1992; Poulos, 1994). En général, la mise en place d'un ensemble de pieux modifie les propriétés du sol de façon différente de celle d'un pieu isolé dont le comportement sert de référence. L'effet de groupe peut, suivant les cas, améliorer ou réduire les qualités mécaniques du sol environnant et, par conséquent, conduire à un accroissement ou à une diminution de la charge nominale du pieu en groupe par rapport à celle du pieu isolé.

L'objectif principal de l'étude entreprise est de fournir des coefficients simples, à appliquer aux courbes de réaction obtenues pour un pieu isolé pour retrouver celles des pieux disposés en groupe. Le programme comporte 40 essais sur des couples de pieux identiques disposés à différents espacements variant de 2 à $8 \mathrm{~B}$ et répartis sur 5 conteneurs. Plusieurs modes et ordres de mise en place des pieux dans le massif de sable ont été testés. Les distances entre pieux sont comptées d'axe en axe $(\mathrm{S}=2 \mathrm{~B}$ correspond donc à un entre-axe de 2B, par exemple).

Cet article présente une partie de cette étude expérimentale et en particulier l'étude de l'effet de groupe sur le comportement d'un couple de pieux chargé latéralement dans un massif sableux et celle de l'influence du mode d'installation dans le sable.

\section{2}

\section{Implantation et mode d'installation des pieux dans le sol}

\section{1}

\section{Instrumentation des pieux}

Les pieux utilisés dans cette campagne d'essais sont des tubes en aluminium AU4G, dont les caractéristiques mécaniques ont été déterminées à partir d'essais de traction conformément à la norme NF-A 03-302. La charge à la limite apparente d'élasticité est de 1065 daN. Tous les essais de chargement latéral ont été menés de manière à éviter la plastification des pieux, en maintenant la contrainte maximale mesurée sur le pieu inférieure à la contrainte élastique admissible.

Les pieux ont une longueur totale de $380 \mathrm{~mm}$, un diamètre externe de $18 \mathrm{~mm}$, une épaisseur de $1,5 \mathrm{~mm}$ et une rigidité de flexion de $201 \mathrm{~N} . \mathrm{m}^{2}$. La fiche dans le sol est fixée à $300 \mathrm{~mm}$. Les essais ont été conduits à une accélération centrifuge de $40 \mathrm{~g}$. Ceci permet donc de simuler des pieux prototypes de $720 \mathrm{~mm}$ de diamètre externe et de $12 \mathrm{~m}$ de fiche. La rigidité dans ce cas est de $514 \mathrm{MN} \cdot \mathrm{m}^{2}$.

Chaque pieu a été équipé de 20 paires de jauges de déformation collées à l'extérieur du pieu sur deux génératrices diamétralement opposées et disposées le long de la fiche suivant un montage demi-pont. L'espace- 
ment entre les jauges est de $15 \mathrm{~mm}$. La mesure des déplacements en tête du pieu est assurée par deux capteurs de déplacement inductif de type HBM de course $\pm 10 \mathrm{~mm}$ situés respectivement aux cotes $65 \mathrm{~mm}$ et $20 \mathrm{~mm}$ par rapport à la surface du sol (DP1, DP2, DP3 et DP4). Un capteur de force avec un montage des jauges en pont complet mesure l'effort latéral appliqué en tête. L'effort latéral est appliqué à l'aide du dispositif de chargement développé au LCPC (Fig. 1) et transmis aux pieux grâce à un câble métallique comme le montre la figure 2. Cette liaison par câble traversant les deux pieux permet d'éviter d'introduire des moments de flexion parasites en tête des pieux comme cela a été relaté dans les travaux de Williams (1979) et Randolph (1981).

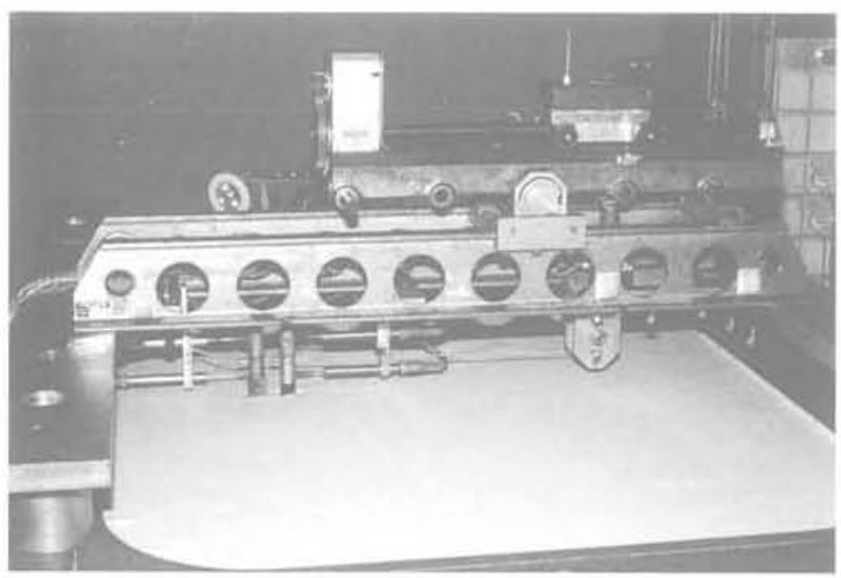

FIG.1 Dispositif de chargement.

Loading device.

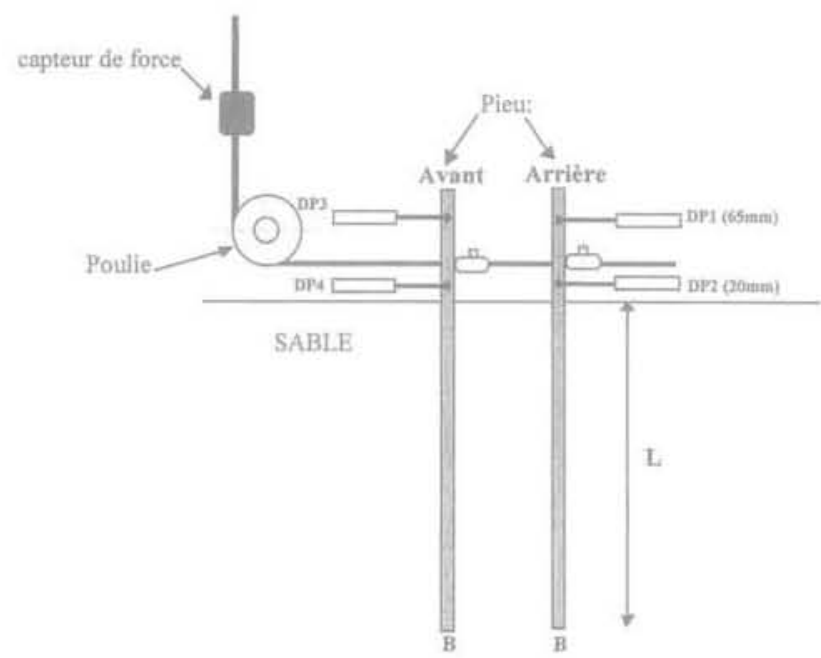

FG. 2 Notation et instrumentation des pieux. Notation and piles equipements. rieur à $1 \%$ (Garnier et al., 1993). Deux densités différentes ont été considérées $\left(16,2 \mathrm{kN} / \mathrm{m}^{3}\right.$ et $\left.15,4 \mathrm{kN} / \mathrm{m}^{3}\right)$ correspondant respectivement à des indices de densité de 0,81 et 0,63 . La densité de mise en place est contrôlée a posteriori à l'ajde de boîtes calibrées placées au sein du massif lors du remplissage (Fig. 3).

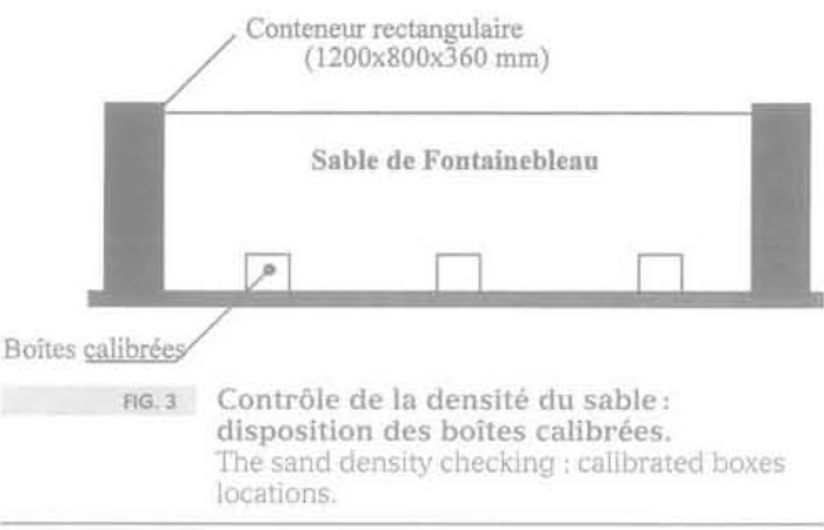

Il s'agit d'un sable fin siliceux propre dont le diamètre moyen des grains est de $200 \mathrm{~mm}$. Pour le modèle des pieux retenus, le rapport entre le diamètre du pieu et celui des grains est d' environ 90 donc très supérieur à la valeur minimale de 40 habituellement admise pour éviter les effets d'échelle (Ovesen, 1979). Une vérification de la bonne homogénéité du massif de sable est réalisée en cours de rotation à l'aide des essais au minipénétromètre statique (Fig.4).

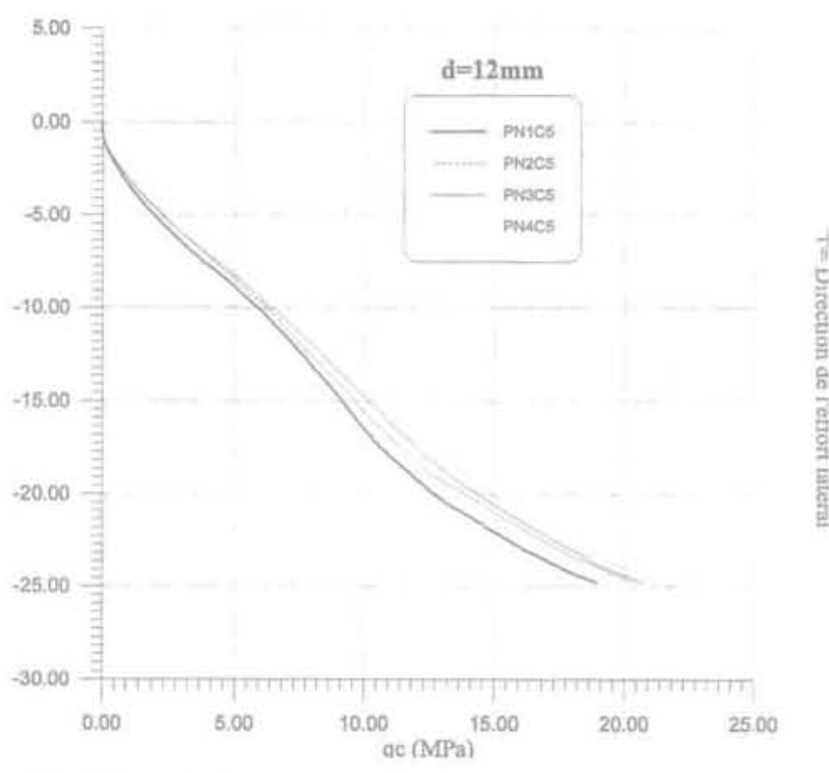

FiG.4 Mesures pénétrométriques en macrogravité sous accélération de $40 \mathrm{~g}$. CPT test data at glevel.

\section{Massif de sol}

Le massif de sable est constitué de sable fin de Fontainebleau blanc déposé dans les conteneurs par pluviation à l'aide la trémie automatique, Ce système de remplissage permet de confectionner un massif de sable homogène, avec des écarts sur les densités infé-

\section{Mode d'installation des pieux dans le massif sableux}

Plusieurs modes de mise en place des pieux ont été utilisés:

- pluviation du sable autour du pieu, ce qui peut être 
assimilé à l'installation du pieu dans un forage (sans refoulement de sol);

- battage avec refoulement de sol;

- vérinage avec refoulement de sol.

Ces modes de mise en place différents, ont pour but d'étudier les deux effets de la disposition des pieux en groupe, qui peuvent parfois se cumuler:

- effet de la mise en place des pieux qui peut faire évoluer les caractéristiques mécaniques du terrain (cas des pieux avec refoulement);

- effet purement géométrique où les déformations et les contraintes induites dans le sol par l'un des pieux, peuvent influencer la réaction du sol sur les autres pieux du groupe. Cet effet peut se manifester, que l'installation soit faite avec ou sans refoulement et, dans le premier cas, il peut se superposer à l'effet précédent.

Le refoulement est obtenu par fermeture des pieux à leur base. Si les pieux forés ne posent pas le problème de l'ordre d'installation, il n'en est pas de même pour les pieux battus ou vérinés. De ce fait, l'ordre de mise en place des pieux du couple est également étudié en inversant cette opération sur le pieu avant et arrière du couple.

Les paramètres étudiés sont:

- la distance entre les pieux du couple : $2 \mathrm{~B}$ à $8 \mathrm{~B}$;

- le mode de mise en ceuvre (avec ou sans refoulement),

- Iordre de mise en cuvre (pour les pieux avec refoulement).

Les deux pieux utilisés dans le couple sont récupérés, après chaque essai, pour être réutilisés dans les groupes suivants. Un exemple d'implantation des pieux dans le conteneur est montré sur la figure 5. Afin d'éviter les interactions, les pieux ont été disposés tels que la distance entre les bords du conteneur et entre chaque zone d'essai soit supérieure à 10B.

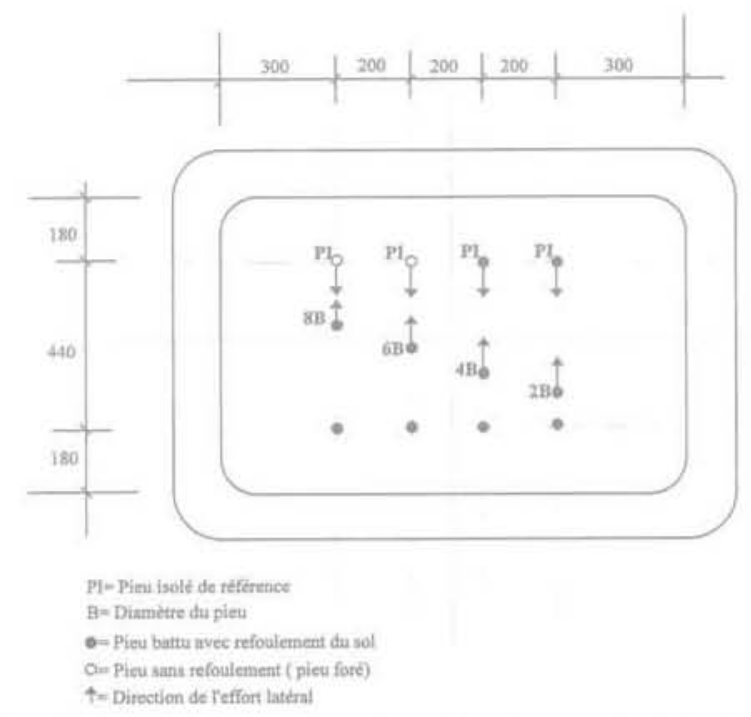

FG.5 Disposition des pieux du couple dans le conteneur.

Coupes piles location in the container.

Le battage des pieux est effectué à $1 \mathrm{~g}$ à l'aide du dispositif de battage spécial du LCPC. La masse utilisée pour le battage pèse $695 \mathrm{~g}$ et sa hauteur de chute est de $470 \mathrm{~mm}$. Le nombre de coups nécessaire à la pénétration totale varie suivant le poids volumique du sable (environ 80 coups pour un poids volumique de $16,2 \mathrm{kN} / \mathrm{m}^{3}$ et seulement 50 coups pour un poids volumique du sable de $15,4 \mathrm{kN} / \mathrm{m}^{3}$ ). Le vérinage des pieux est opéré à l'aide d'un vérin hydraulique à commande automatique. L'enfoncement des pieux est réalisé avec une vitesse faible d'environ $1 \mathrm{~mm} / \mathrm{s}$. Ce vérin est équipé d'un capteur de force permettant de mesurer la résistance à l'enfoncement et d'un capteur de déplacement mesurant l'enfoncement des pieux dans le sol. Cet enfoncement des pieux est suivi directement sur l'écran d'un ordinateur ce qui permet d'arrêter l'opération à la fiche choisie $(300 \mathrm{~mm})$ et aussi de détecter toute anomalie survenant sur le pieu au cours de cet enfoncement. Un exemple de ces courbes d'enfoncement des pieux est représenté sur la figure 6 (q étant la force d'enfoncement du pieu dans le massif par sa section: $\mathrm{F} / \mathrm{S}_{\text {pieu }}$.

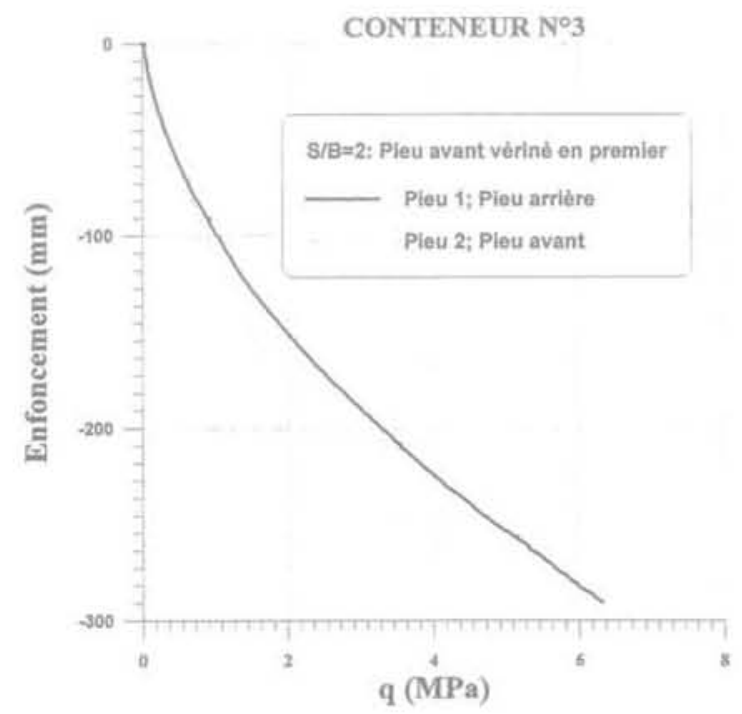

HG. 6 Courbes d'enfoncement du couple de pieux $\left(\gamma=16,2 \mathrm{kN} / \mathrm{m}^{3}, \mathrm{~S} / \mathrm{B}=2\right.$, valeurs modèles).

Driving curves of piles couples $\left(\gamma=16,2 \mathrm{kN} / \mathrm{m}^{2}\right.$, $\mathrm{S} / \mathrm{B}=2$, models data).

Ces courbes nous ont permis d'une part, de confirmer par leur répétitivité, la bonne homogénéité du massif reconstitué par pluviation et, d'autre part, de mettre en évidence l'existence d'une interaction entre les pieux du couple lors du fonçage. Le fonçage du second pieu nécessite en effet des efforts plus grands que ceux du premier. On peut indiquer que la distance limite observée de cette interaction est de l'ordre de 3B à $4 \mathrm{~B}$ en rappelant toutefois que ces opérations sont conduits à $1 \mathrm{~g}$.

Par ailleurs des essais de couples de pieux et ceux des pieux isolés de référence ont été doublés d'un conteneur à l'autre dans le but de confirmer leur répétitivité et la concordance d'un conteneur à l'autre. La figure 7 donne un exemple de ces vérifications réalisées sur les pieux de référence.

\section{3}

\section{Influence de l'espacement entre les pieux sur le comportement du groupe}



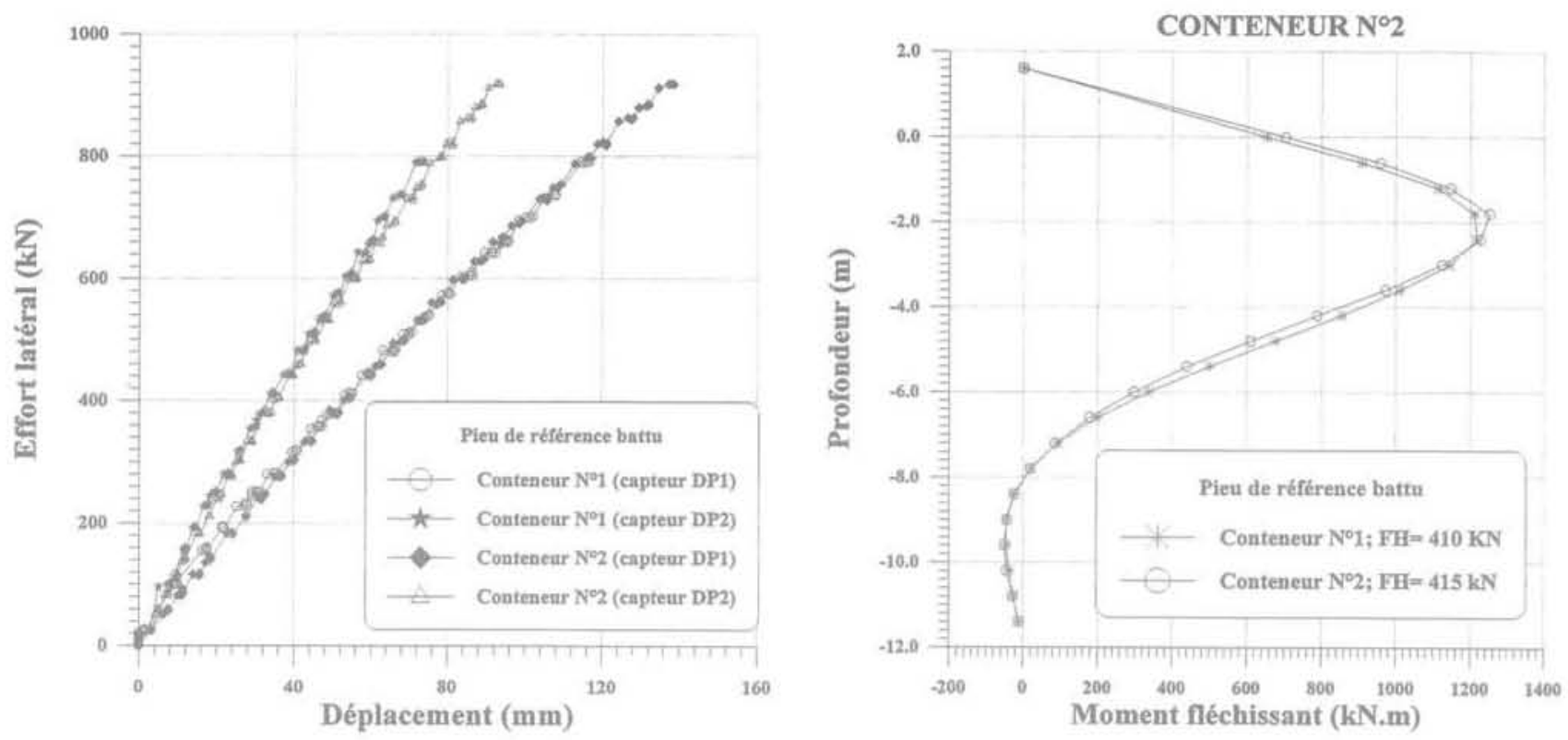

FG. 7 Comparaison des déplacements en tête et des moments fléchissants mesurés sur les pieux de référence 1 et 2 du conteneur $N^{\circ} 5 ; \gamma=16,2 \mathrm{kN} / \mathrm{m}^{3}$ ).

Piles references top displacements and bending moments comparison (Container $\mathrm{N}^{\circ} 5, \gamma=16,2 \mathrm{kN} / \mathrm{m}^{3}$ ),

\section{1}

\section{Déplacement en tête et effort repris par le groupe}

\section{1 .5}

\section{Pieux vérinés}

Les courbes de chargement de la figure 8 montrent que, pour ce type de pieux, l'espacement a peu d'influence sur leur comportement.

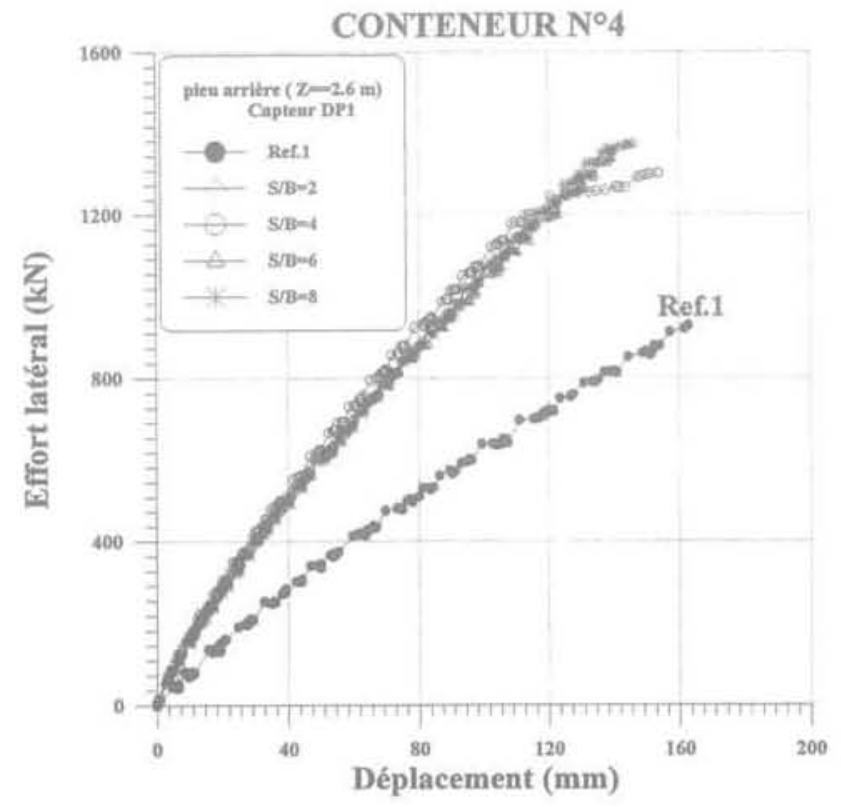

Du fait du mode de liaison entre les deux pieux (câble métallique), il était important de vérifier que les deux pieux êtaient sollicités en même temps. La figure 9 montre qu'en effet les déplacements des pieux avant et arrière sont presque confondus.

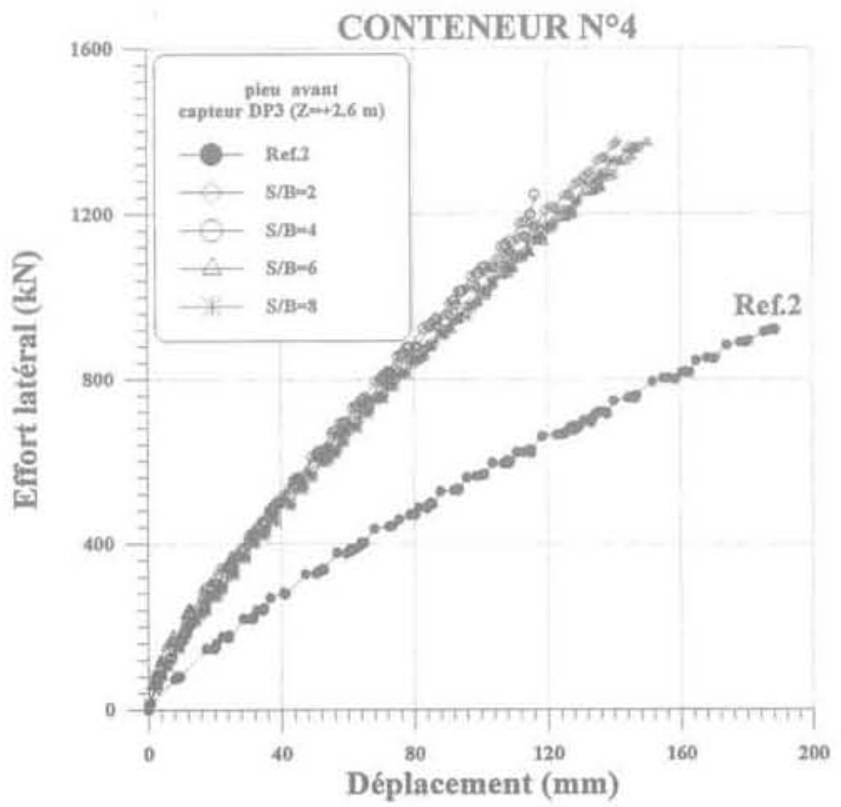

FGG.8 Influence de l'espacement sur les déplacements en tête des pieux vérinés (effort total sur les couples et effort sur le pieu de référence isolé, $\left.\gamma=16,2 \mathrm{kN} / \mathrm{m}^{3}\right)$.

Spacing influence on jacked piles top displacements (total lateral load on the couple and lateral load on the reference single, $\gamma=16,2 \mathrm{kN} / \mathrm{m}^{3}$. 

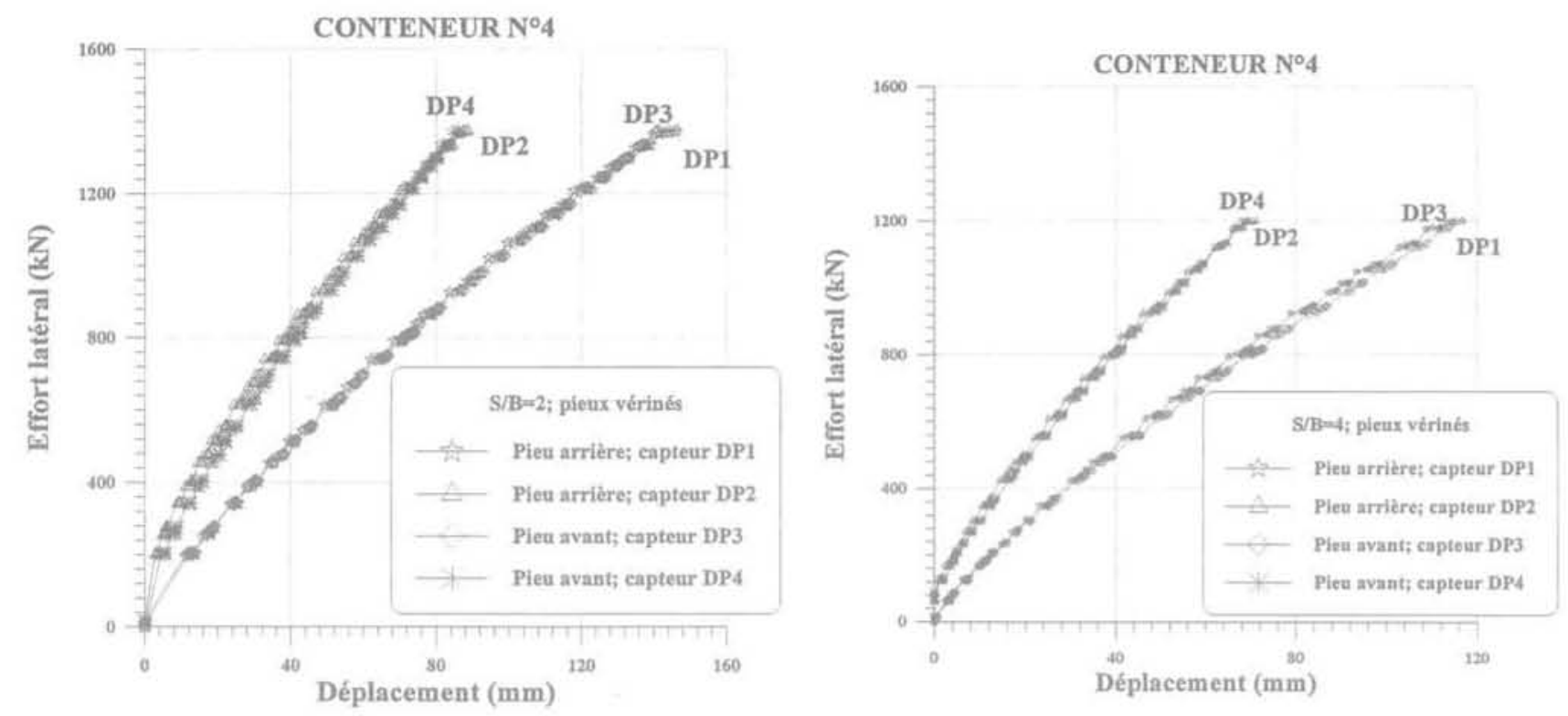

FG.9 Comparaison des déplacements en tête du pieu avant et du pieu arrière du groupe (pieu vériné, $\gamma=16,2 \mathrm{kN} / \mathrm{m}^{3}$ ).

Comparison of top displacements of the front and rear pile of the group (jacked pile, $\gamma=6,2 \mathrm{kN} / \mathrm{m}^{2}$ ).

Une méthode simple pour évaluer l'effet de groupe est de calculer son coefficient d'efficacité définie comme suit:

$$
\mathrm{e}=\frac{\begin{array}{l}
\text { charge latérale sur le groupe } \\
\text { pour un déplacement donné }
\end{array}}{\begin{array}{l}
\mathrm{n} \times \text { charge latérale sur le pieu } \\
\text { isolé à un même déplacement }
\end{array}} \times 100
$$

où n est le nombre de pieux dans le groupe.

Une valeur de $e=100 \%$, indique qu'il n'y a pas interaction entre les pieux. Leur comportement n'est pas différent de celui d'un pieu isolé. Pour les pieux étudiés ici, l'efficacité moyenne du groupe a été déterminée pour des déplacements inférieurs ou égaux à 10\% du diamètre $\mathrm{B}$ des pieux correspondant à des sollicitations de service. Le tableau I regroupe les résultats obtenus à partir des valeurs de déplacement enregistrés sur le capteur DP2 situé à $20 \mathrm{~mm}$ par rapport à la surface du sol.

Ce tableau confirme les constatations faites sur les courbes de chargement. Il n'est pas mis en évidence, sur ce type de pieux, d'effet de groupe significatif, même pour des distances entre pieux très faibles ( $2 B$ et $4 \mathrm{~B})$. A moins de $8 \%$ près, les charges reprises par les couples, pour un déplacement en tête donné, sont le double de celles du pieu isolé.

Ce résultat, qui peut surprendre, se trouve confirmé par les essais sur les pieux battus, eux aussi mis en place avec refoulement du sol.

\section{1 .9}

\section{Pieux battus}

La figure 10 montre à titre d'exemple les courbes de chargement obtenues sur les pieux du conteneur $n^{\circ} 2$ (pieu isolé référence et couples). On y observe que les courbes relatives aux couples sont très voisines, quelle que soit la distance entre les deux pieux (2B à 8B). Elles montrent des charges très proches du double de celles du pieu de référence.
TABLEAUT Efficacité « "e du groupe de pieux. Group efficiency " $e$ ".

\begin{tabular}{c|c|c|c}
\hline $\begin{array}{c}\text { Espacement } \\
\text { relatif S/B }\end{array}$ & $\begin{array}{c}\text { Pieux vérinés } \\
\mathrm{e}(\%)\end{array}$ & $\begin{array}{c}\text { Pieux battus } \\
\mathrm{e}(\%)\end{array}$ & $\begin{array}{c}\text { Pieux forés } \\
\mathrm{e}(\%)\end{array}$ \\
\hline 2 & 101,3 & 98,4 & 72,0 \\
\hline 4 & 97,7 & 104,9 & 87,0 \\
\hline 6 & 91,6 & 112,2 & 96,0 \\
\hline 8 & 95,2 & 104,6 & 99,0 \\
\hline
\end{tabular}

Comme dans le cas des pieux vérinés, on a vérifié que les deux pieux du couple étaient bien sollicités en mème temps. Le calcul des coefficients d'efficacité du groupe effectué dans ce cas confirme que le comportement des pieux du couple est proche de celui du pieu isolé, comme le montre le tableau I. On remarque cependant que les coefficients d'efficacité obtenus ici, présentent des valeurs parfois légèrement supérieures à $100 \%$. Cette constatation a déjà été signalée par Williams (1979) lors des essais sur des modèles de groupe de pieux chargès latéralement dans le sable et confirmée par Randolph (1981). D'après ces auteurs, cela peut être dû au système d'attache transmettant les charges aux pieux du groupe qui limiterait la rotation en tête des pieux du groupe. Le moment fléchissant résistant qui en résulte a été estimé par Williams (1979) à environ $20 \%$ du moment fléchissant au niveau de la surface du sable.

Cette explication n'est probablement pas satisfaisante ici puisque le même câble métallique et les mêmes liaisons sont utilisés sur les couples et sur les pieux isolés. Ce câble ne transmet pas de moment et ne gène donc pas la rotation des pieux. On peut par contre penser que cet effet de groupe positif est du au mode de mise en place qui améliore les caractéristiques mécaniques autour des pieux du couple.

On retiendra donc que les pieux mis en place avec refoulement de sol (battus ou vérinés) ne présentent pas d'effet de groupe significatif, dans des sables à forte densité. 

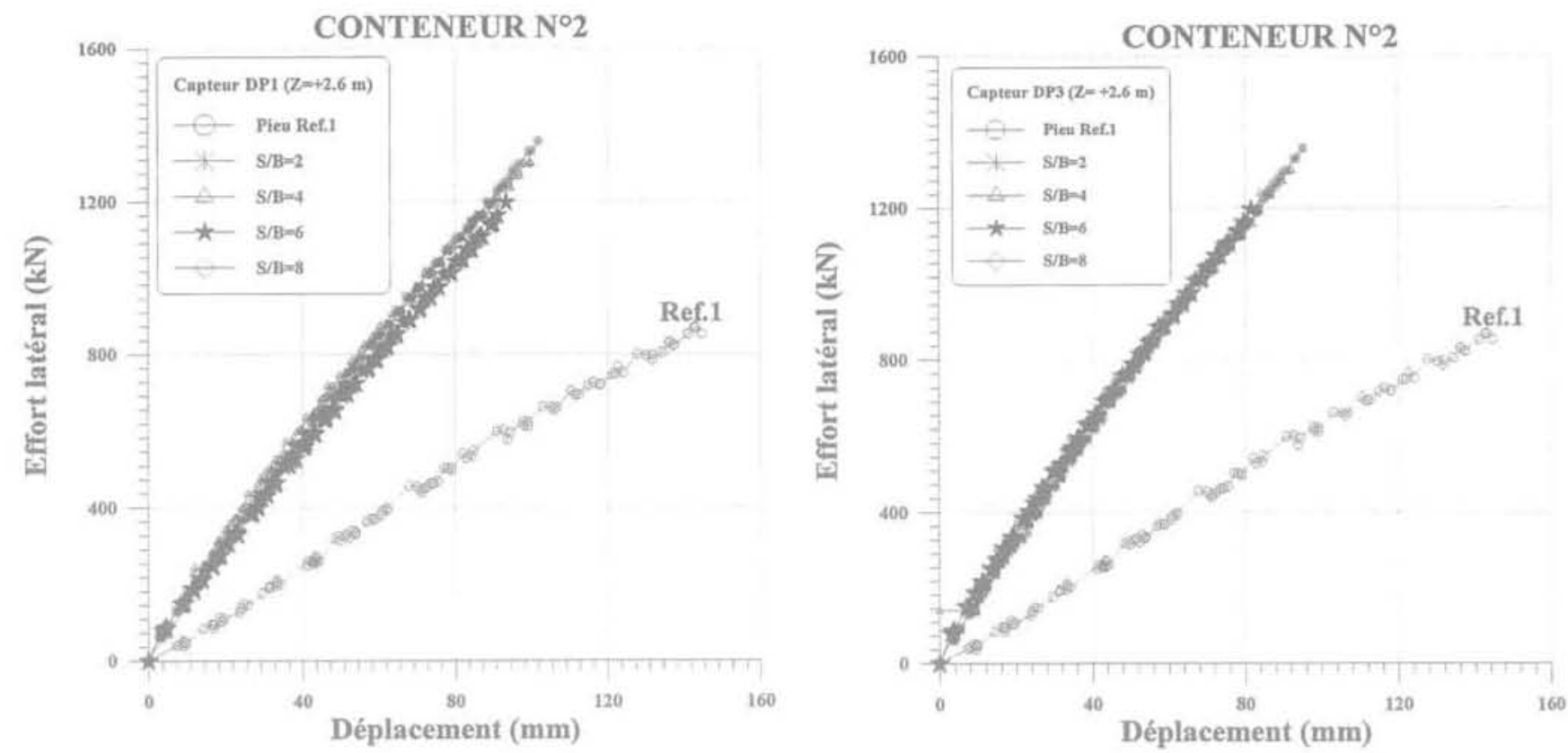

FiG. 10 Influence de l'espacement sur le déplacement en tête des pieux battus (effort total sur les couples et effort sur le pieu de référence isolé, $\gamma=16,2 \mathrm{kN} / \mathrm{m}^{3}$.

Spacing influence on driven top piles displacements (total lateral load on the couple and lateral load on the reference single pile, $\left.g=16,2 \mathrm{kN} / \mathrm{m}^{3}\right)$.

Comme on le verra plus loin, cela ne signifie pas que les deux pieux du couple reprennent les mêmes efforts, égaux à ceux du pieu isolé.

\section{1 .7}

\section{Pieux forés}

Dans le cas des pieux forés, l'influence de l'espacement entre les pieux est par contre nettement visible sur les courbes de chargement comme le montre, la figure 11. La variation du coefficient d'efficacité (tableau I) indique qu'à partir d'un espacement d'environ $8 \mathrm{~B}$, le comportement des pieux du groupe est identique à celui d'un pieu isolé et la charge appliquée en tête du couple, se repartit de la même façon sur les pieux du groupe. Ce résultat confirme ce qui a été déjà rapporté par plusieurs chercheurs (Davisson, 1970; Randolph, 1981; Barton, 1982; Mezazigh, 1990).

\section{2}

\section{Répartition des efforts entre les pieux}

\section{alage \\ Pieux vérinés}

Comme déjà observé pour les déplacements en tête, l'espacement entre les pieux du groupe semble avoir un effet assez faible sur les courbes des moments fléchissants. Sur la figure 12, on présente les moments fléchissants enregistrés sur le pieu avant et le pieu arrière du groupe. Deux remarques peuvent être tirées de ces courbes:

- les moments fléchissants sur le pieu avant et le pieu arrière au niveau de la surface restent très proches. Ceci indique que les deux pieux reprennent le même effort en tête:
- le moment maximal sur le pieu avant et le pieu arrière sont également voisins.

On constate, cependant, que la profondeur des moments maximaux des deux pieux est différente, Ce phénomène pourrait être lié au refoulement du sol causé par l'enfoncement des pieux. Il semble disparaître pour des espacements supérieurs à environ 3 ou

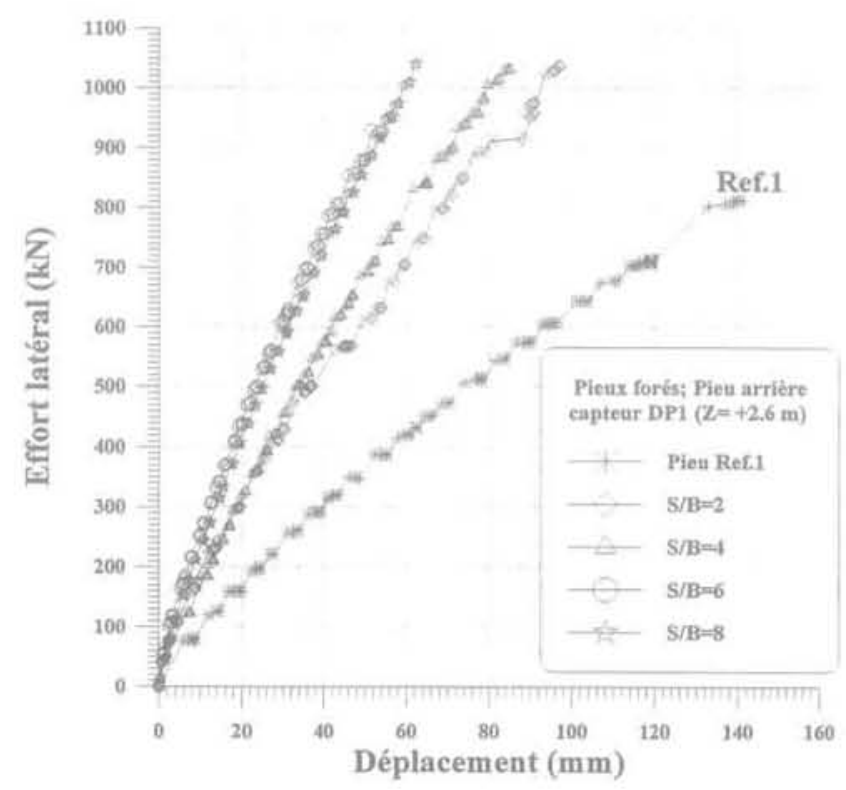

FG. 11 Influence de l'espacement sur les déplacements en tête des pieux forés (effort total sur les couples et effort sur le pieu isolé, $\gamma=16,2 \mathrm{kN} / \mathrm{m}^{3}$.

Spacing influence on bored piles top displacements (total lateral load on the couple and lateral load on the reference single pile, $\gamma=16,2 \mathrm{kN} / \mathrm{m}^{3}$ ). 

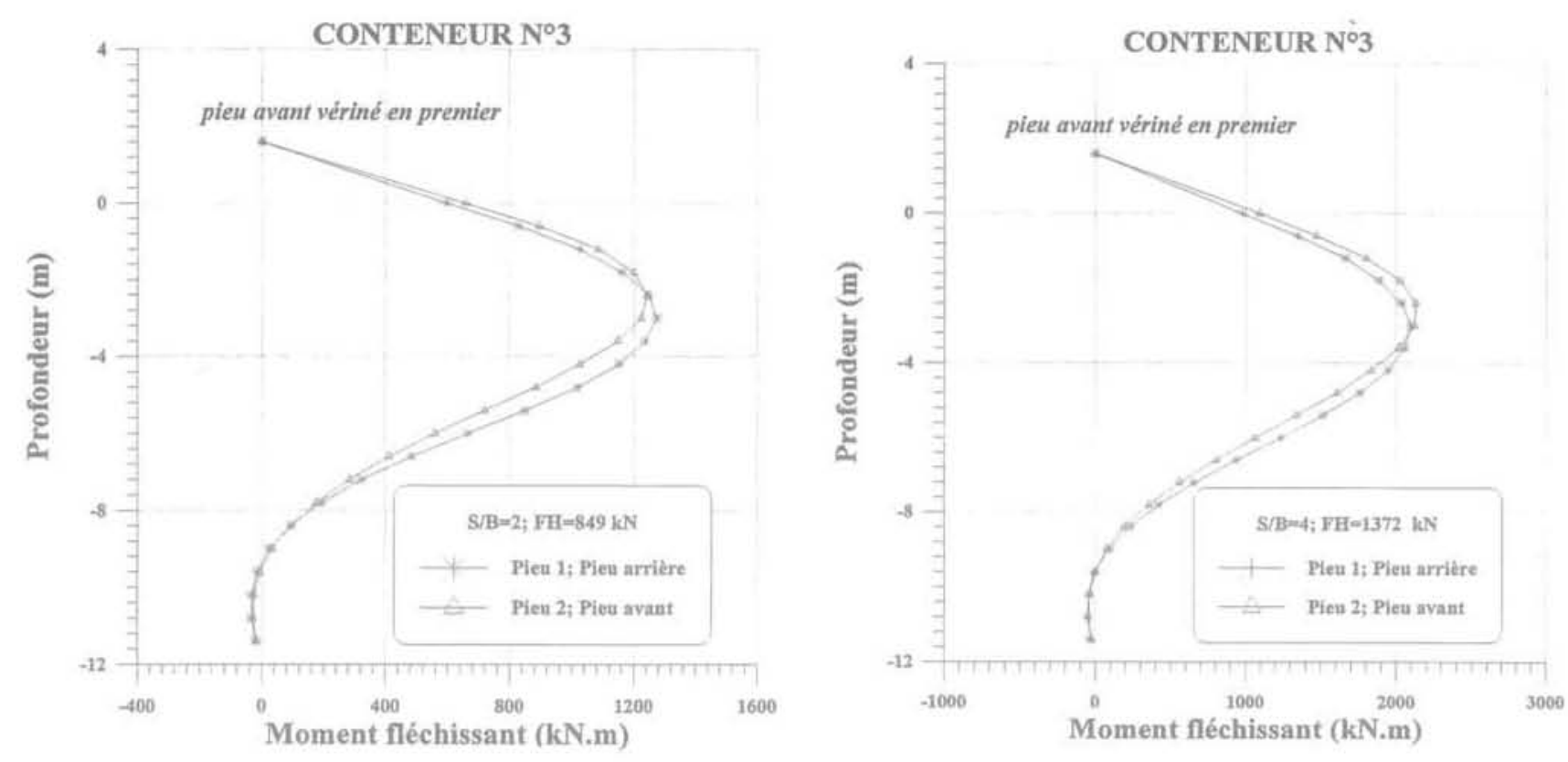

FG.12 Courbes des moments fléchissants des pieux avant vérinés pour deux espacements ( $\mathrm{S} / \mathrm{B}=2$ et $\mathrm{S} / \mathrm{B}=4$, $\left.\gamma=16,2 \mathrm{kN} / \mathrm{m}^{3}\right)$.

Bending moments curves of jacked front piles for two spacings $\left(S / B=2\right.$ and $\left.S / B=4, \gamma=16,2 \mathrm{kN} / \mathrm{m}^{2}\right)$.
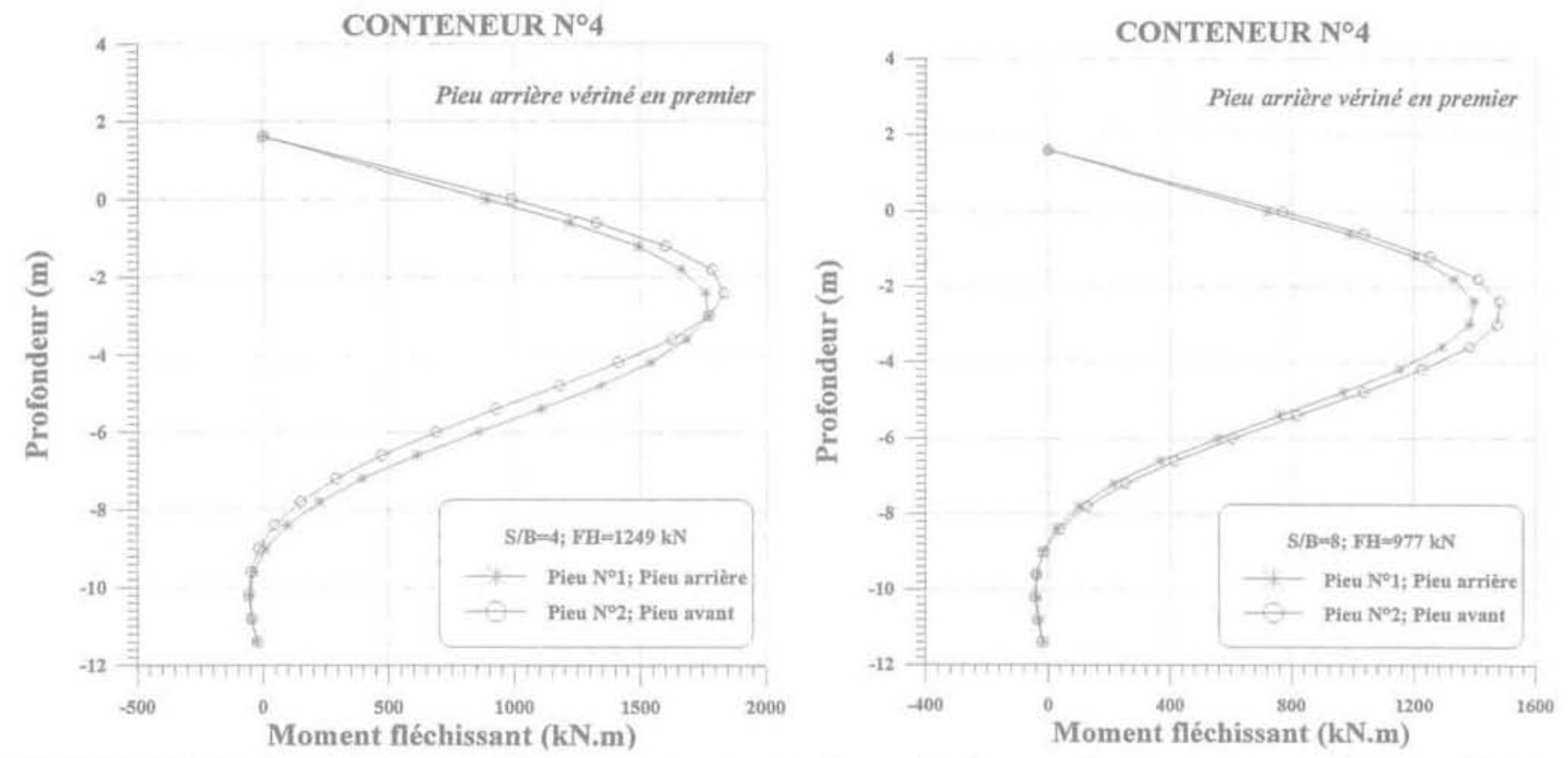

FIG. 13 Courbes des moments fléchissants des pieux arrières vérinés pour deux espacements $(S / B=4$ et $S / B=8$, $\gamma=16,2 \mathrm{kN} / \mathrm{m}^{3}$ ).

Bending moments curves of jacked rear piles for two spacings $\left(\mathrm{S} / \mathrm{B}=4\right.$ and $\left.\mathrm{S} / \mathrm{B}=8, \gamma=16,2 \mathrm{kN} / \mathrm{m}^{3}\right)$.

4B (figure 13). En effet, au cours du vérinage des pieux, on observe sur le pieu déjà en place un léger soulèvement lié au refoulement du sol engendré par l'enfoncement du second pieu. Ce phénomène s'est répété quelle que soit la charge appliquée en tête et quel que soit l'ordre de mise en place des pieux.

\subsection{2}

\section{Pieux battus}

Dans le cas des pieux installés par battage, on constate pour les pieux distants de $2 \mathrm{~B}$ une légère diffé- 
rence de comportement entre le pieu arrière et le pieu avant, comme on peut le voir sur la figure 14. En effet, à cet espacement, on note qu'au niveau de la surface le pieu du couple présente des moments fléchissants environ $20 \%$ fois plus importants que sur le pieu arrière. En outre, le point de moment maximal est situé à une profondeur plus faible sur le pieu avant, ce qui avait aussi été observé sur les pieux vérinés. Cet effet disparaît rapidement dès l'espacement de 4B. On signale par ailleurs, que ces observations ont été faites pour tous les incréments de chargement, indépendamment de l'ordre de mise en place des pieux.
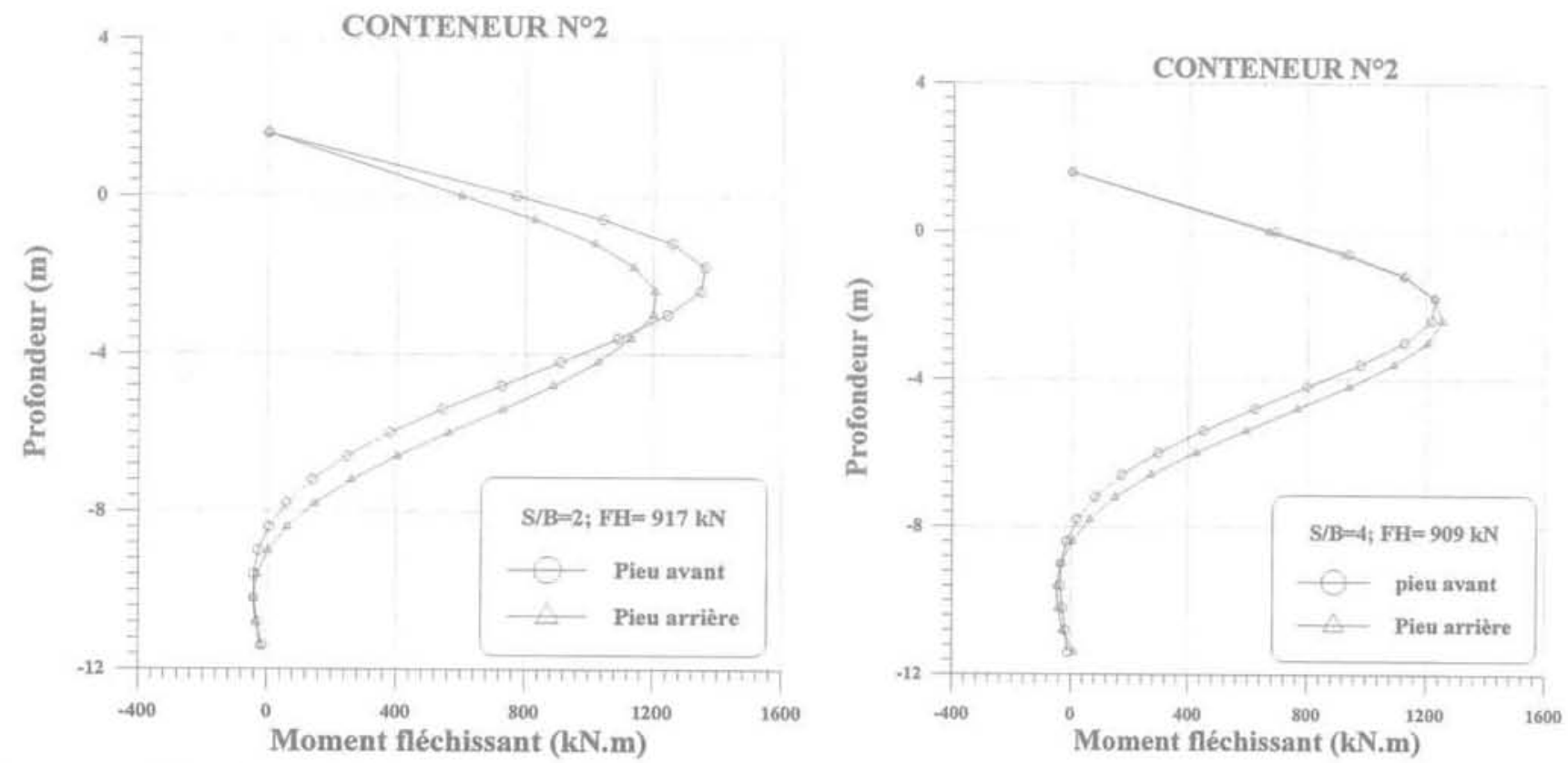

FG. 14 Influence de l'espacement sur le comportement des pieux battus (cas : $S / B=2,4$ ). Spacing influence on driven piles behavior (case: $S / B=2,4$ ).

\section{3.:2.9.}

\section{Pieu forés}

L'analyse des moments fléchissants mesurés sur les pieux forés montre une influence plus marquée de l'espacement entre les pieux sur leur comportement

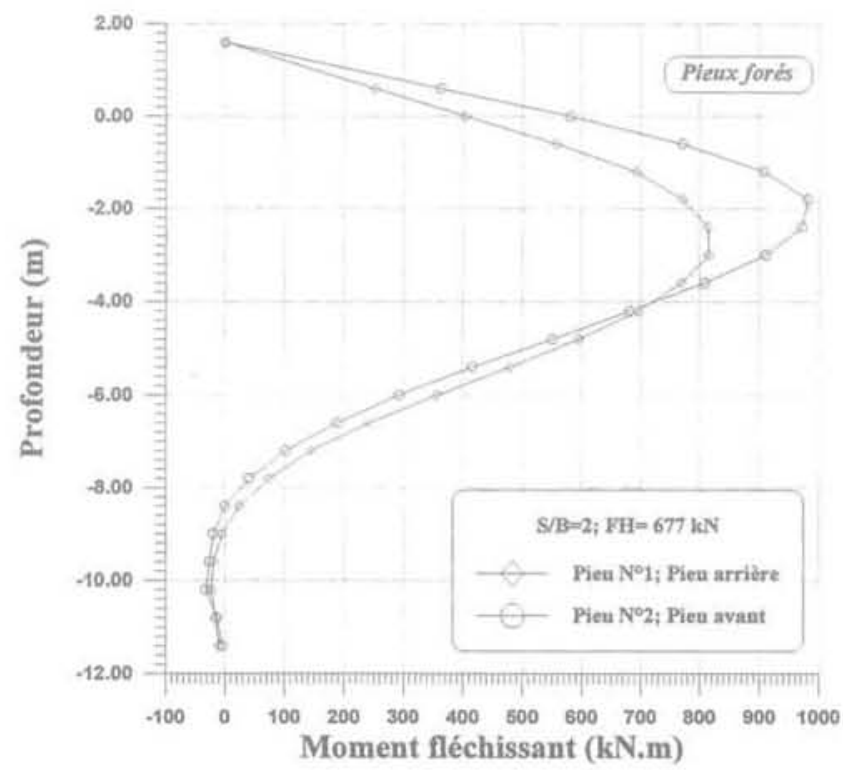

sous charge latérale. Les figures 15 et 16 donnent des exemples de l'influence de l'espacement sur les moments fléchissants des deux pieux du groupe.

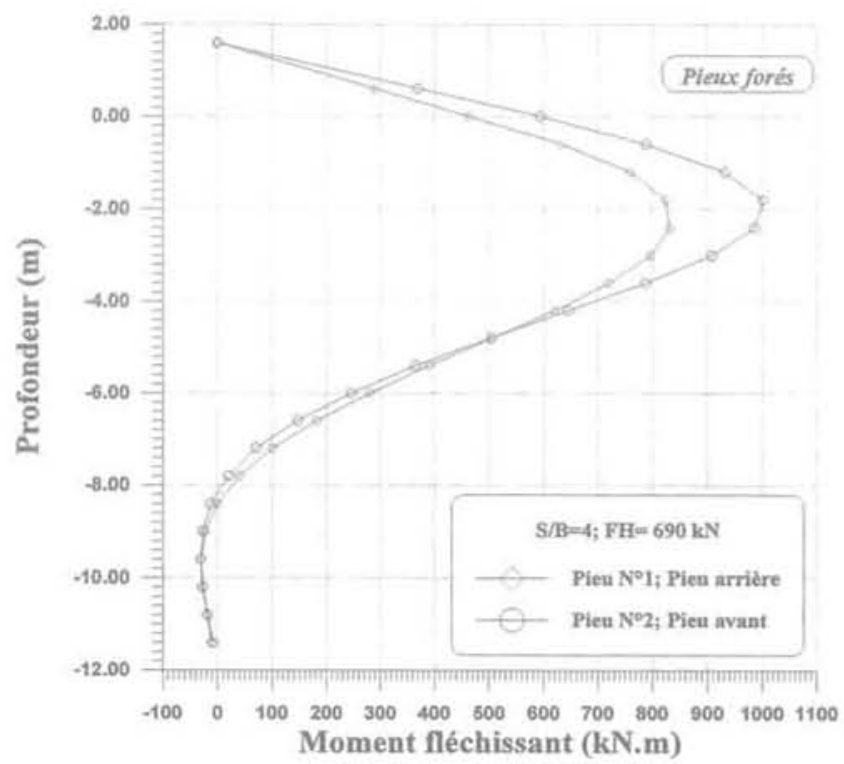

BQ. 15. Influence de l'espacement sur les courbes des moments fléchissants des pieux forés ( $\mathrm{S} / \mathrm{B}=2 ; \mathrm{S} / \mathrm{B}=4$; $\gamma=16,2 \mathrm{kN} / \mathrm{m}^{3}$.

Spacing influence on bending moments curves of bored piles $\left(\mathrm{S} / \mathrm{B}=2 ; \mathrm{S} / \mathrm{B}=4 ; \gamma=16,2 \mathrm{kN} / \mathrm{m}^{3}\right)$. 

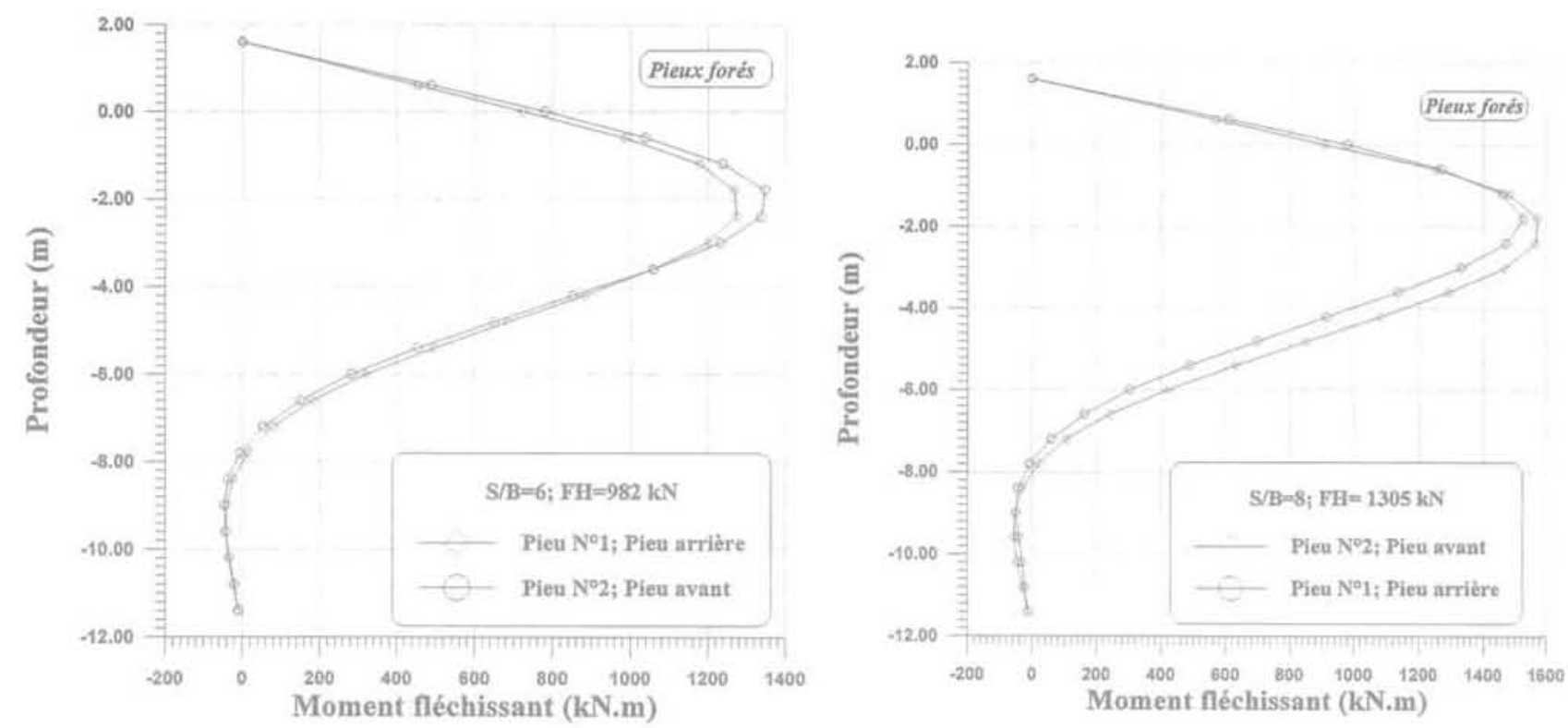

FIG. 16

Influence de l'espacement sur les courbes des moments fléchissants des pieux forés $(\mathrm{S} / \mathrm{B}=6 ; \mathrm{S} / \mathrm{B}=8$; $\gamma=16,2 \mathrm{kN} / \mathrm{m}^{3}$ ).

Spacing influence on bending moments curves of bored piles $\left(\mathrm{S} / \mathrm{B}=6 ; \mathrm{S} / \mathrm{B}=8 ; \gamma=16,2 \mathrm{kN} / \mathrm{m}^{2}\right)$.

Cette influence se manifeste d'abord sur la répartition de la charge latérale appliquée en tête. En effet, pour les faibles espacements on constate que le pieu avant du couple reprend une plus large part de l'effort latéral total appliqué en tête. Ainsi, le pourcentage de l'effort repris par le pieu avant de l'ordre de $60 \%$ pour un espacement de 2B. La répartition de la charge s'équilibre entre les deux pieux du couple au fur et à mesure que leur espacement augmente et atteint $50 \%$ sur chacun des pieux à partir d'un espacement de $8 \mathrm{~B}$ comme on le constate sur le tableau II et les figures 15 et 16 .
Par ailleurs, on note également une influence de l'espacement sur les moments maxima ainsi que sur leur position en profondeur. En effet, le moment maximum mesuré sur le pieu avant à des espacements de $2 \mathrm{~B}$ et $4 \mathrm{~B}$ sont environ $20 \%$ fois supérieur à ceux du pieu arrière du couple. Par contre, la position du moment maximum est plus profonde sur les pieux arrières pour les faibles espacements ( $2 \mathrm{~B}$ et $4 \mathrm{~B}$ ) mais elle est la même sur les deux pieux pour $\mathrm{S}=8 \mathrm{~B}$ comme l'indique le tableau III et le montrent les exemples des figures 15 et 16 .

TABLEAu II Répartition de la charge totale sur les deux pieux du couple. Total load distribution on the two piles of couple.

\begin{tabular}{c|c|c}
\hline Espacement relatif S/B & $\begin{array}{c}\text { Taux de la charge reprise } \\
\text { par le pieu AVANT (\%) }\end{array}$ & $\begin{array}{c}\text { Taux de la charge reprise } \\
\text { par le pieu ARRIERE (\%) }\end{array}$ \\
\hline 2 & 60 & 40 \\
\hline 4 & 56 & 44 \\
\hline 6 & 52 & 48 \\
\hline 8 & 50 & 50 \\
\hline
\end{tabular}

tABleau III Influence de l'espacement sur la profondeur du moment maximum des pieux du groupe. L désigne la fíche des pieux dans le sol.

Spacing effect on the maximum bending moment depth. $\mathrm{L}$ is the embedded length of pile in the soil.

\begin{tabular}{c|c|c}
\hline Espacement relatif S/B & $\begin{array}{c}\text { Position du moment max. } \\
\text { Pieu avant }\end{array}$ & $\begin{array}{c}\text { Position du moment max. } \\
\text { Pieu arriere }\end{array}$ \\
\hline 2 & $0,16^{*} \mathrm{~L}$ & $0,26^{*} \mathrm{~L}$ \\
\hline 4 & $0,16^{*} \mathrm{~L}$ & $0,21^{*} \mathrm{~L}$ \\
\hline 6 & $0,16^{*} \mathrm{~L}$ & $0,21^{*} \mathrm{~L}$ \\
\hline 8 & $0,16^{*} \mathrm{~L}$ & $0,16^{*} \mathrm{~L}$ \\
\hline
\end{tabular}




\section{Influence de l'ordre de mise en place des pieux}

Les courbes de la figure 17 présentent les résultats d'essais sur des couples de pieux vérinés et de pieux battus où l'ordre de mise en place des pieux a été inversé.

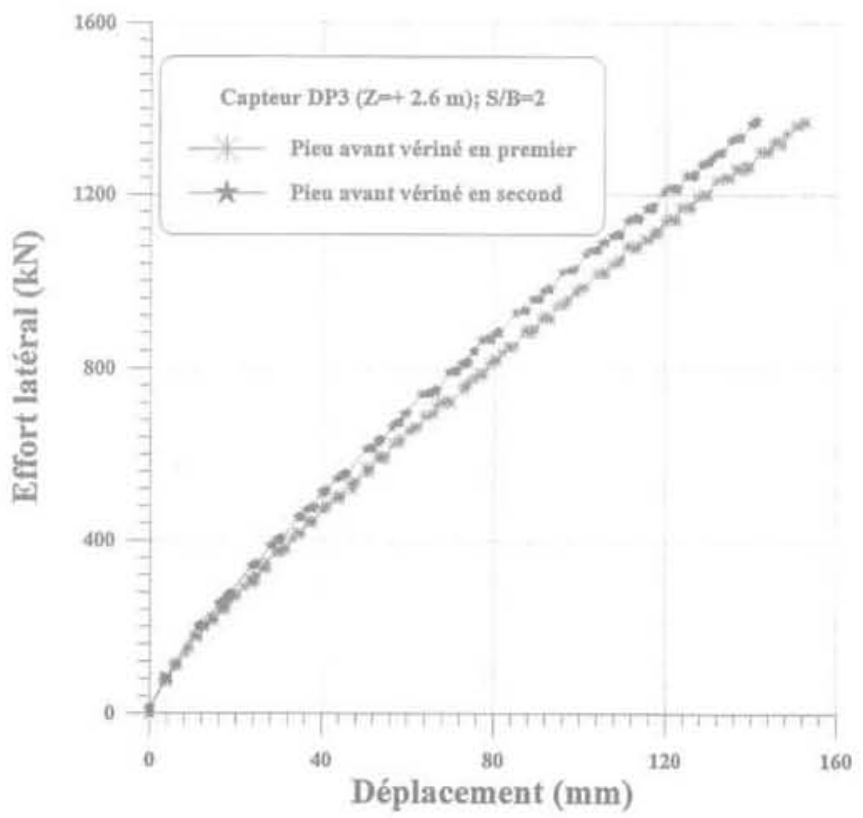

Les réponses des couples sont très peu affectées par l'ordre de mise en place. Les écarts entre les courbes de chargement sont dans la fourchette des dispersions normales des résultats. Les mêmes constations ont été faites sur les pieux pour les autres espacements. On peut donc en conclure que l'effet de groupe sur des couples de pieux ne dépend pas de l'ordre de mise en place des deux pieux.

FG. 17 Influence de l'ordre de mise en place sur les déplacements en tête des pieux $\left(\mathrm{S} / \mathrm{B}=2, \gamma=16,2 \mathrm{kN} / \mathrm{m}^{3}\right.$, pieux vérinés et pieux battus). Influence of the installation order on piles top displacements ( $\mathrm{S} / \mathrm{B}=2, \gamma=16.2 \mathrm{kN} / \mathrm{m}^{3}$, jacked and driven piles).

\section{Conclusion}

L'influence de plusieurs paramètres sur le cornportement du couple de pieux disposés en ligne a été étudiée en utilisant toujours les mêmes pieux et le même sable.

Pour les pieux sans refoulement (pieux forés), les résultats des essais montrent clairement l'influence de l'espacement sur le comportement des pieux. Les conclusions suivantes peuvent être tirées dans le cas des pieux alignés suivant la direction d'application de la charge:

- le couple de pieux fléchit davantage que le pieu isolé pour un chargement similaire moyen par pieu; en d'autres termes, pour un déplacement donné, la charge latérale reprise par le couple avec un espacement de 2B ne représentent que $70 \%$ de celle que peuvent reprendre deux pieux isolés. Il faut, un espacement d'au moins $8 \mathrm{~B}$ pour que ce rapport atteigne la valeur 1 indiquant donc la disparition de l'effet de groupe : - la distribution de la charge dans les pieux du groupe n'est pas uniforme. Les pieux avant reprennent une part plus importante de la charge que les pieux arrières. A un espacement de $2 \mathrm{~B}$, ce rapport est d'environ $60 \%$ pour le pieu avant et de $40 \%$ pour le pieu arrière. A partir de $8 \mathrm{~B}$, les deux pieux supportent le même effort latéral;

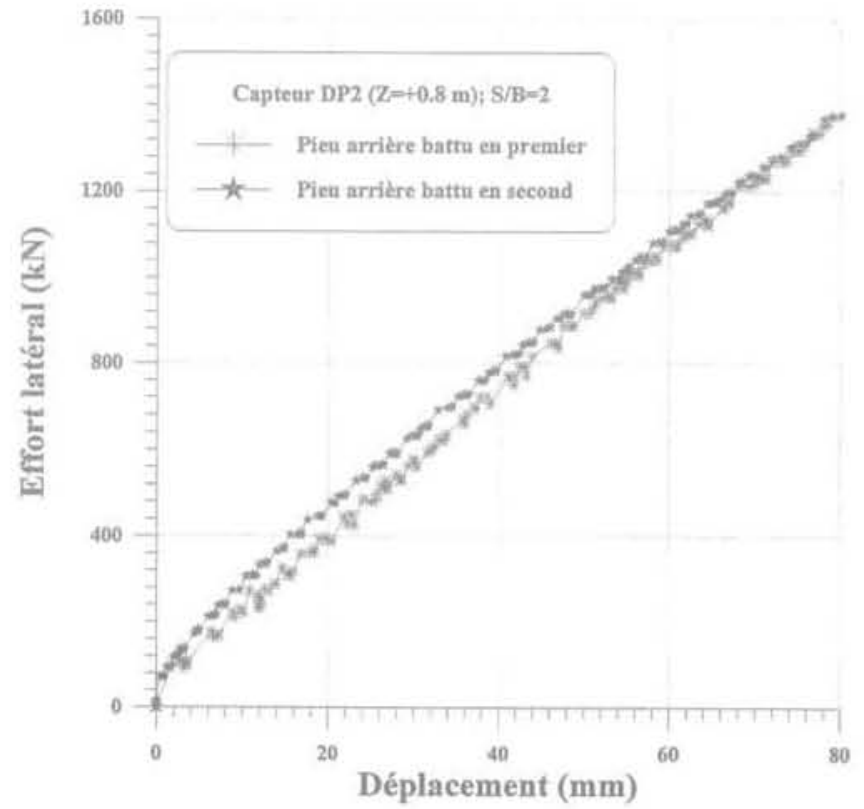


les pieux forés (sans refoulement) et l'effet positif lié à leur mode de mise en place.

Il convient cependant de rappeler que les pieux ont été mis en place à $1 \mathrm{~g}$, avant la centrifugation des modèles et que les essais n'ont porté que sur des massifs de sable.

L'effet de groupe positif observé sur certains couples de pieux battus pourrait se révéler encore plus important si les contraintes géostatiques avaient été simulées pendant la mise en place des pieux. Ces battages et fonçages en cours de centrifugation seront bientôt possibles grâce au téléopérateur embarquable à $100 \mathrm{~g}$ mis en service.

Les travaux en cours portent sur l'étude directe de l'effet de groupe sur les courbes de réaction P-Y qu'il est possible de déterminer expérimentalement à l'aide de pieux instrumentés. Les premiers résultats indiquant pour les faibles espacements, une nette réduction des pressions sur les pieux arrières. Les essais prévus sur les groupes de 4 et 9 pieux permettront par ailleurs de généraliser les résultats obtenus sur les couples.

\section{Bibliographie}

[1] Barton Y.O, - "Laterally loaded model piles in sand. Centrifuge tests and finite element analysis $\mathrm{n} . \mathrm{PhD}$, University of Cambridge, $1982,182 \mathrm{p}$.

[2] Brown D.. Morrison C.. Reese L. "Lateral load behaviour of pile group in sand $x$. ASCE, yol. 114, $\mathrm{n}^{\circ} 11,1988$, p. 1261-1277

[3] Cooke R.W., Price G. Tarr K. - $\alpha$ Jacked piles in London Clay: a study of load transfer and settlement under working conditions D. Géotechnique 29, n², 1979, p. 113-147.

[4] Garnier J., Kus B., Levacher D. - "Cartographie des densités de massifs de sable reconstitués par pluviation $\mathrm{n}$. $6^{\circ}$ Colloque franco-polonais de Mécanique des Sols, Douai, 1993, p. 105-112.

[5] Garnier J. - $\alpha$ Modèles réduits en mécanique des sols $»$. AUGC, École Centrale de Nantes, 18 mai 1995, p. 21-44.

[6] Haahr F., Hansen B. - « Group effect for laterally loade piles in sand $n$. Proc of XIt International Conference on Soil Mechanics and Foundation Engineering
(ICSMFE), Rio de Janeiro, vol.1, 1989, p. 427-432

[7] José L., Sayed M. - "Group efficiency of piles driven into sands: A simple approach 1. Geotechnical Engineering Congress, vol. I, 1991, p. 346-355.

[8] Levacher D, - "Groupe de pieux sollicités latéralement: Analyse et réflexions en vue d'une étude paramétrique en centrifugeuse ». Rapport interne LCPC, MEC, $n^{\circ} 89.08,1989,29 \mathrm{p}$.

[9] Levacher D., Mezazigh S., Vasslin E. Sevin J. - «Instrumentation et comportement de pieux d'accostage sous faible charges horizontales ». IVe Journées nationales Génie côtier-Génie civil. Dinard, 17-19 avril, 1996; p. 379-407.

[10] Mezazigh S. - Comportement d'un couple de pieux sollicité latéralement: étude paramétrique $»$. Mérnoire de DEA. ENSM Nantes, 1990, 88p.

[11] Mezazigh S., Levacher D., Garnier J. "Comportement d'un couple de pieux sollicité latéralement \%. Ites Journées inter-universitaires, Génie côtier, Le
Havre, 1990, p. 149-160.

[12] Mezazigh S, - $\alpha$ Etude expérimentale des pieux chargés latėralement: proximité d'un talus et effet de groupen. Mémoire de thèse de doctorat de l'université de Nantes et de l'École Centrale de Nantes, 1995, 217p.

[13] Ovesen N.K. - a The scaling law relationship. Panel discussiony. $7^{e}$ ECSMFE, Brighton, vol.4, 1979, p.319323.

[14] Poulos H.G. - «Effect of pile driving on adjacent piles in clay 刃. Canadian Geotechnical Journal, vol.31, 1994, p.856-867.

[15] Randolph M. - "The response of flexible piles to lateral loading 1. Canadian Geotechnical Journal, vol.3.1, n², 1981, p. 733-751.

[16] Shublaq E.W. - « Soil disturbance sue to installation of model piles and pile groups x. Soils and Fundations vol.32, $n^{\circ} 4,1992$, p. 17-26.

[17] Williams D.J. - « The behaviour of model piles in dense sand $x$. PhD thesis, University of Cambridge, 1979. 